\title{
Frühe Antibiotikakuren erhöhen Ekzem- und Heuschnupfen-Risiko
}

\begin{abstract}
Kinder, die in den ersten beiden Lebensjahren antibiotisch behandelt wurden, haben ein erhöhtes Risiko für allergische Ekzeme und Heuschnupfen.
\end{abstract}

Den Ergebnissen einer neuen, beim europäischen Lungenkongress ERS 2016 vorgestellten Metaanalyse zufolge sollten Antibiotika bei Babys und Kleinkindern im Alter unter zwei Jahren nicht leichtfertig eingesetzt werden. Die Autorengruppe um Dr. Fariba

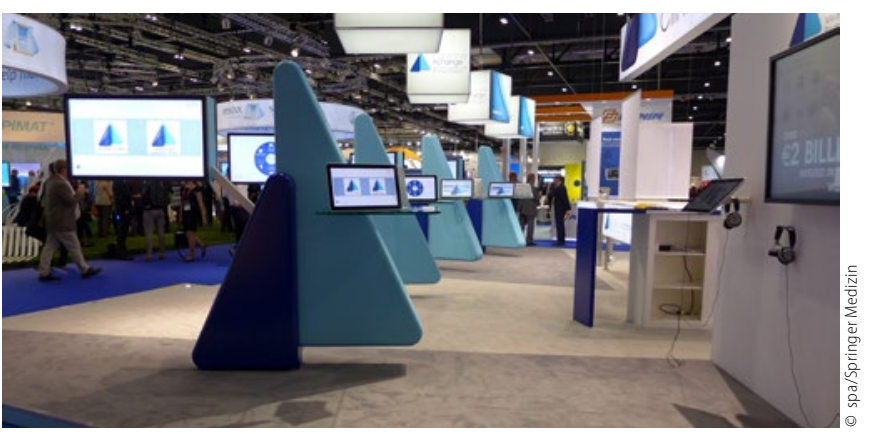

Stilisierte Heuschnupfennasen beim ERS-Kongress. Antibiotika beeinflussen das Darmmikrobiom und so wohl das Allergierisiko.
Ahmadizar von der Universität in Utrecht hatte sämtliche Studien zwischen 1966 und 2015 analysiert, welche die Auswirkungen einer frühen Antibiotikatherapie und späteren Erkrankungen an Ekzemen oder Heuschnupfen untersuchten.

Sie fanden 22 Studien mit 395.000 Patienten, die die Assoziation mit Ekzem-Erkrankungen testeten. Kinder mit Antibiotikabehandlung innerhalb der ersten zwei Lebensjahre wiesen ein um 15\% (cross-sektionale Studien) bzw. um 41\% (Kohortenstudien) erhöhtes Risiko auf, an Ekzemen zu erkranken.

Zum Zusammenhang mit allergischer Rhinitis fanden die Autoren ebenfalls 22 Studien mit 257.000 Patienten. Das Heuschnupfen-Risiko erhöhte sich um 14\% (cross-sektionale Studien) bzw. 56\% (Kohortenstudien), wenn die Kinder in den ersten Jahren antibiotisch behandelt worden waren.

Die Assoziation war sowohl bezüglich Ekzem als auch bezüglich Heuschnupfen deutlicher, wenn die Kinder mehr als eine Antibiotikakur erhalten hatten. Die Autoren vermuten, dass Antibiotika durch die Veränderung des intestinalen Mikrobioms Änderungen im Immunsystem verursachen, welche das Risiko für die allergischen Erkrankungen erhöhen.

Dr. med. Dirk Einecke

Quelle: International Congress 2016 der European Respiratory Society (ERS), London, 3.-7. September 2016

\section{Stellen Computer genauere Diagnosen als die Doktoren?}

\begin{abstract}
Mithilfe von „künstlicher Intelligenz" lassen sich chronische Lungenerkrankungen akkurater diagnostizieren und unnötige Zusatzuntersuchungen vermeiden, behaupteten Autoren der Universität Leuven in Belgien beim europäischen Lungenkongress ERS 2016.
\end{abstract}

Diagnostik von Atemwegserkrankungen ist ein mühseliges Geschäft: Beschwerden des Patienten sowie Befunde von Spirometrie, Bodyplethysmografie und Diffusionstest müssen interpretiert und unter einen Hut gebracht werden. Selbst mit fachärztlicher Expertise kann dies schwierig sein, ohne diese sind Fehlern Tür und Tor geöffnet. Erinnert sei nur an die weit verbreitete Unsicherheit, auch nur zwischen Asthma und COPD sauber zu differenzieren.

Ein Autorenteam aus Leuven in Belgien hat nun einen Versuch unternommen, mithilfe künstlicher Intelligenz den menschlichen diagnostischen Irrtum weitgehend zu minimieren. Sie haben dazu 968 Personen erstmals einer kompletten Lungenfunktionstestung unterzogen, ggf. wurden weiterfüh- rende diagnostische Maßnahmen wie CT oder EKG gemacht und auf dieser Basis eine Diagnose gestellt. Diese wurde, wie es hieß, von einer großen Gruppe klinischer Experten validiert.

Anschließend wurde ein algorithmischer Prozess entwickelt, der sowohl anamnestische und klinische Daten als auch die Ergebnisse der Untersuchungsergebnisse berücksichtigt und einen Computer in die Lage versetzt, eine prädiktive Datenanalyse zur Diagnosefindung vorzunehmen. Das Konzept besteht darin, dass die Maschine dabei lernt, erklärte Studienautor Wim Janssens.

Tatsächlich konnten die Belgier nachweisen, dass die computergestützten Diagnosen genauer waren als die ärztlichen. Der Computer gehe standardisierter und objektiver vor, erklärte Janssens. Die Autoren versprechen sich von ihrem Programm zumindest, weniger erfahrenen Kollegen eine Hilfestellung anbieten zu können, zudem eine Zeitersparnis und eine Vermeidung unnötiger Zusatzuntersuchungen.

Dr. med. Dirk Einecke

Quelle: International Congress 2016 der European Respiratory Society ERS, London, 3.-7. September 2016 